\title{
New Aspects of Neurotransmitter Release and Exocytosis: Rho-Kinase-Dependent Myristoylated Alanine-Rich C-Kinase Substrate Phosphorylation and Regulation of Neurofilament Structure in Neuronal Cells
}

\author{
Yasuharu Sasaki* \\ Department of Pharmacology, Pharmaceutical Science, Kitasato University, \\ 5-9-1, Shirokane, Minato-ku, Tokyo 108-8641, Japan
}

Received May 2, 2003; Accepted June 27, 2003

\begin{abstract}
Myristoylated alanine-rich C-kinase substrate (MARCKS) is an actin-binding protein whose function may be regulated by the phosphorylation of multiple sites, in which the phosphorylation site domain (PSD) is recognized to have three or four PKC-dependent sites. Recently, it is considered that MARCKS is implicated in some neuronal functions, such as synaptic vesicle trafficking and neurotransmitter release, through regulation of the actin-containing cytoskeletal structure; this is based on the experimental results with short-term or prolonged pretreatment with phorbol esters and treatment by protein kinase $\mathrm{C}$ (PKC) inhibitor. However, the precise molecular mechanism is yet obscure. Recently, we have demonstrated that MARCKS is phosphorylated at Ser159 in PSD by Rho-kinase in vitro and that the phosphorylation occurred in neuronal cells upon stimulation with lysophosphatidic acid (LPA), and its phosphorylation was inhibited by a novel and specific Rho-kinase inhibitor, H-1152. Our results allow us to speculate that a preinflammatory substance, such as LPA, interleukin 1- $\beta$, and bradykinin, augments MARCKS phosphorylation in a novel signal transduction pathway besides the PKC-involved one, and thereby induces the release of a neurotransmitter through a reorganization of actincontaining microfilaments at the cell periphery, the so-called "active zone". In this section, I address a novel mechanism for MARCKS phosphorylation and its related cellular function.
\end{abstract}

Keywords: Rho-kinase, lysophosphatidic acid, phosphorylation, actin structure

\section{Introduction}

Neurotransmitter release is mediated by synaptic vesicle exocytosis at the active zone of synaptic nerve terminals. A small number of these vesicles, which are reserved at the active zone, are docked with the synaptic membrane to release neurotransmitters upon such a depolarization, leading to presynaptic $\mathrm{Ca}^{2+}$ entry, while the majority of vesicles are anchored at neurofilament structures at an area away from the active zone (1). The former is referred to as the readily releasable vesicle and the latter as the reserved vesicles. Upon a stimulation, the reserved vesicles are released from the neurofilaments mainly consisting of F-actin microfilaments

*Corresponding author. FAX: +81-3-3442-3875

E-mail: sasakiy@pharm.kitasato-u.ac.jp and subsequently traffic to the active zone. The active zone and the neighboring sites contain F-actin meshworks, which act as a barrier that rejects the access of the vesicles to the active zone. Thus, disassembly of F-actin meshworks may be necessary to facilitate the trafficking of the vesicles. The dis-anchoring and trafficking of the vesicles seem to be involved in several types of protein phosphorylations, but a large part of the molecular mechanism remains obscure, or in part controversial.

Several kinds of stimuli, which include thrombin, lysophosphatidic acid (LPA), bradykinin, and phorbol ester (12-O-tetradecanoylphorbol 13-acetate, TPA; phorbol 12,13-dibutyrate, $\mathrm{PDBu}$ ), increase the pool size of releasable vesicles in cultured cells, or in part evoke the release of neurotransmitter but not fully $(2-5)$. However, such a stimuli-involved mechanism is pre- 
cisely unknown. Recently, Lonart and Sudhof reported that PDBu enhances the releasable pool size of glutamate and norepinephrine from a synaptosome preparation, but did not evoke the release; however, the mechanism for the events should be resolved (6). In particular, the target molecule of protein kinase $\mathrm{C}$ (PKC) and also its involved pathway(s) in vesicle disanchoring and trafficking were obscure. On the other hand, it is demonstrated that PKC-dependent phosphorylation of myristoylated alanine-rich $\mathrm{C}$ kinase substrate (MARCKS) occurs coincidently with F-actin disassembly and potentiation of catecholamine release in a permeabilized chromaffin cells (7). Overexpression of MARCKS is reported to decrease catecholamine release from $\mathrm{K}^{+}$-stimulated human neuroblastoma (8). These results seem to be attributed to MARCKS-dependent reorganization of F-actin structure, which may allow the vesicles to access the presynaptic membrane and its adjacent area, the so-called "active zone". Thus, the dis-anchoring and trafficking of synaptic vesicles may require cytoskeleton dynamics (disorganization and reorganization), in particular F-actin containing neurofilament structure in the active zone (9). However, the molecular mechanism of other stimuli besides PKC activators on neurotransmitter release remain obscure. Although thrombin-evoked serotonin release from platelets seems to be associated with MARCKS phosphorylation (5), it is unknown what kind(s) of protein kinase catalyze it, PKC or another kinase.

Recently, we reported an LPA-evoked phosphorylation of MARCKS at Ser159, which is recognized as the PKC site, in human neuroteratoma cells; this phosphorylation is suppressed by a Rho-kinase-specific inhibitor, H1152 (10, 11). In this session, we would address the mechanism of LPA-induced neurotransmitter release, focusing on the phosphorylation of MARCKS at the phosphorylation site domain (so-called PSD, characterized as PKC-dependent sites) by Rhokinase.

\section{LPA-evoked phosphorylation of MARCKS}

There are many reports that LPA can enhance, or in part, evoke the release of neurotransmitter release from neuronal cells or synaptosome preparation, but the explanation for the intracellular mechanism remains controversial $(12-14)$. LPA could recruit at least 3 pathways to induce cellular events including cell proliferation, contraction, and exocytosis, and the receptor of which is coupled with trimeric $\mathrm{G}$ protein and activates intracellular signaling pathways of phospholipase $\mathrm{C}$ (PLC)/PKC, Ras/mitogen-activated protein (MAP)kinase, and Rho/Rho-kinase $(12,15)$. As MARCKS may be involved in neurotransmitter release, we tried to determine whether or not LPA stimulation can evoke the phosphorylation of MARCKS and what kinase(s) is activated by LPA in neuronal cells, PKA, PKC, or others. We detected the phosphorylation in human neuroteratoma (NT-2) cells stimulated with LPA, which were determined with phosphorylation site-specific antibodies; one recognizes the Ser144-phospho-MARCKS and another, Ser159-phospho-MARCKS. The former site is considered as a MAP-kinase-site and the latter as a PKC-site, respectively $(16,17)$. LPA evoked Ser159 phosphorylation but not Ser144 phosphorylation in neuronal cells in culture (Fig. 1). Interestingly, the adjacent site of Ser159 possesses a sequence of ${ }^{153} \mathrm{KKKKXXSXX}^{161}$ that is recognized as a Rho-kinase site as well as a PKC site. Rho-kinase can phosphorylate several proteins that contain a basic amino acid residue at a -2 or more $\mathrm{N}$-terminal-side position from the phosphorylation site; ${ }^{651} \mathrm{RRST}^{654}$ in the myosin binding subunit of phosphatase (MBS $130 \mathrm{kDa})(18),{ }^{36} \mathrm{RVT}^{38}$ in CPI-17 (19), ${ }^{16}$ RATS $^{19}$ in the myosin light chain (20). With an in vitro system, we confirmed that Ser159 phosphorylation is catalyzed by PKC and Rho-kinase (Fig. 2). Moreover, protein kinase A was confirmed to phosphorylate the Ser159 as well as PKC and Rho-


Fig. 1. MARCKS phosphorylation in NT-2 cells treated with $\mathrm{PDBu}$ or LPA. MARCKS phosphorylation was determined in an immunoblot analysis with an antibody against Ser159-phosphoMARCKS. Note that LPA phosphorylation is quickly responsive and transient while $\mathrm{PDBu}$ phosphorylation is slowly responsive and sustained. Neither LPA-induced nor PDBu-induced phosphorylation bands were reacted with antibody against Ser144-phosphoMARCKS. A: Representative immunoblot. B: Time course of MARCKS phosphorylation. Reproduced from Ref. 11 with permission from Elsevier. 




Fig. 2. Phosphorylation of recombinant human MARCKS by various protein kinases. All the protein kinases tested incorporate ${ }^{32} \mathrm{P}$ into MARCKS, but Cdc5 and MAP-kinase can not phosphorylate the Ser159 in MARCKS. Reproduced from Ref. 11 with permission from Elsevier.

kinase. Taken together with these evidences, it is possible that Ser159 in MARCKS is phosphorylated by Rhokinase.

Ser159 residue in the PSD of MARCKS is phosphorylated by Rho-kinase in neuronal cells

We have developed a highly potent and much more specific inhibitor of Rho-kinase, H1152. Recently, many researchers have recognized that traditional Rho-kinase inhibitors, HA-1077 and Y-27632, are lesser inhibitors of Rho-kinase in several systems. The characteristics of H1152 are summarized in Table 1. We made use of H1152 to determine whether the Ser 159 phosphorylation is catalyzed by Rho-kinase in the cells stimulated with LPA. As shown in Fig. 3, H1152 predominantly inhib-
Table 1. $\mathrm{K}_{\mathrm{i}}$ values of Rho-kinase inhibitors for Ser/Thr protein kinases

\begin{tabular}{lccc}
\hline & \multicolumn{3}{c}{$\mathrm{K}_{\mathrm{i}}(\mu \mathrm{M})$} \\
\cline { 2 - 4 } Protein kinase & $\mathrm{H}-1152$ & $\mathrm{HA}-1077$ & $\mathrm{Y}-27632$ \\
\hline Rho kinase & 0.0016 & 0.33 & 0.14 \\
PKA & 0.63 & 1.0 & 25 \\
PKC & 9.27 & 9.3 & 26 \\
MLCK & 10.1 & 55 & $>250$ \\
PAK & $>100$ & $>100$ & $>100$ \\
\hline
\end{tabular}

MLCK, myosin light chain kinase; PAK, p21-activated kinase.

ited the phosphorylation of MARCKS in NT-2 cells stimulated with LPA, but the PKC activator PDBu did not. Taken together with other concepts that LPA activates $\mathrm{G}_{12 / 13}$-coupled receptor (LPA2, Edg4) in nerve tissue and consequently exerts the Rho/Rho-kinase pathway (see recent reviews 12, 15), our results raise the possibility that MARCKS at Ser159 is alternatively phosphorylated by PKC and Rho-kinase. In other words, the preinflammatory substance LPA recruits the Rhokinase pathway to phosphorylate MARCKS in neuronal cells, which is a novel mechanism for MARCKS phosphorylation.

\section{MARCKS controls F-actin meshwork structure in the active zone of neuronal cell or presynaps}

At the present time, it is well accepted that the phosphorylation of PSD in MARCKS is exclusively catalyzed by $\mathrm{PKC}$ and consequently decreases the interac-
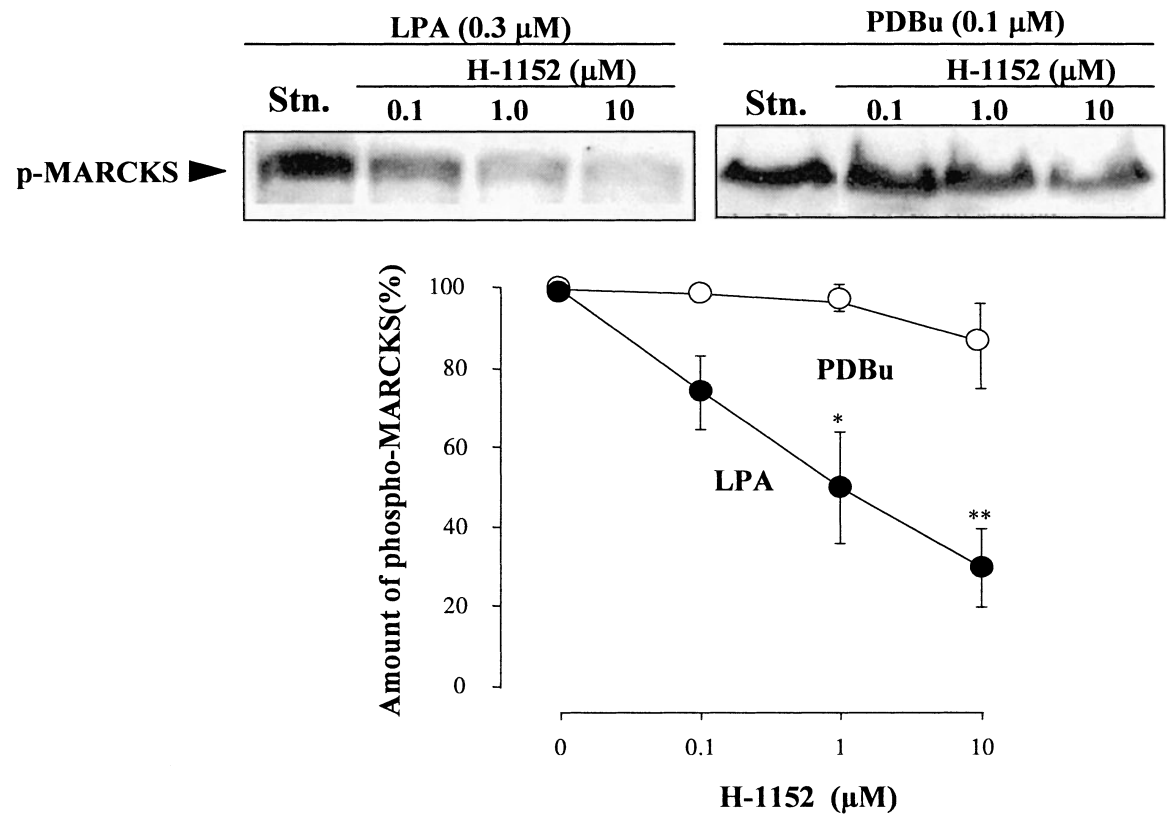

Fig. 3. Inhibition by Rho-kinase inhibitor H-1152 of LPA-induced MARCKS phosphorylation in NT-2 cells. H-1152 scarcely inhibits $\mathrm{PDBu}$-induced phosphorylation. LPA: $0.3 \mu \mathrm{M}$. PDBu: $0.1 \mu \mathrm{M}$. Stn. means the fraction of PKC-phosphorylated MARCKS as a positive standard. $* P<0.05$ and $* * P<0.01$, compared with cells without $\mathrm{H}-1152$ treatment. 
tion activity of the molecule with acidic and hydrophobic molecules such as F-actin, membrane phospholipids, and calmodulin $(21-25)$. It is also known that MARCKS is localized at the presynaptic membrane and its adjacent sites and participates in presynaptic membrane activities, although these molecular mechanisms are not fully elucidated (26). Ohmori et al. elegantly demonstrated using green fluorescent protein (GFP)-MARCKS fusion protein in living cells that MARCKS molecules are localized at the plasma membrane site at a resting state, while upon stimulation with phorbol ester TPA, the molecules are diffused into the cytosol (27). It has been reported that MARCKS interacts with phosphatidilinositol 4,5-bisphosphate $\left(\mathrm{PIP}_{2}\right)$ and its interaction is reduced by the phosphorylation of PSD in the MARCKS molecule (28). As PIP is localized at the inner site of the plasma membrane and regulates the dynamics of $\mathrm{F}$-actin structures at the cell periphery, it is plausible that the F-actin cross-linking activity of MARCKS participates in the dynamics of cortical actin meshworks. Recently, it has been considered that MARCKS regulates neurotransmitter release through reorganization of the F-actin meshwork in an active zone of presynaps $(8,23,24-$ 32 ). In other words, the phosphorylation of the molecule facilitates the approach of synaptic vesicles to the active zone just under the presynaptic membranes.

\section{MARCKS is a candidate for a regulatory component of neurotransmitter release}

There are many reports that the release of neurotransmitter in brain preparations, isolated synaptosomal preparation, and cultured neuronal cells are potentiated by short-term pretreatment with phorbol esters $(32-35)$, while PKC inhibitors and down-regulation of PKC attenuate the enhancement (36). Large fractions of the chromaffin vesicle seem to be excluded from the site near the membrane upon a stimulation with phorbol ester, concomitantly accompanied with disassembly of cortical F-actin structures $(7,29)$. These reactions including MARCKS phosphorylation, disassembly of cortical F-actin structure, and the potentiation of $\mathrm{Ca}^{2+}$ evoked secretion are reported to be inhibited by a synthetic peptide of the PSD of MARCKS. In SH-SY5Y cells, overexpression of MARCKS leads to a decrease in the $\mathrm{K}^{+}$-evoked noradrenaline release (8). Although it is well-known that MARCKS phosphorylation responds to several kinds of stimuli, it is not plausible that all these stimuli activate PKC to phosphorylate PSD. Actually, Neltner et al. have proposed that thrombin evokes a PKC-independent phosphorylation of MARCKS (37). As mentioned above, we demonstrated that the neurotransmitter releasing agonist LPA evokes Rho-kinase-dependent phosphorylation of MARCKS concurrently with reorganization of F-actin structures (Fig. 4). This may raise the possibility that several kinds of stimulants activate multiple protein kinase pathways including PKC and Rho-kinase to phosphorylate the MARCKS at Ser159 and subsequently evoke or enhance neurotransmitter release.

\section{Future perspectives in neurotransmitter release}

The process of neurotransmitter release involves several and sequential steps, among which vesicle trafficking may require reorganization of cortical F-actin meshworks in the active zone and/or near the site. It is likely that LPA alternatively activates Rho and PLC through LPA receptors consequently to activate Rhokinase and PKC, respectively (Fig. 5). Our abovementioned results allow us to speculate that preinflammatory substance LPA-induced activation of Rho-kinase and/or PKC evokes the MARCKS phosphorylation
A

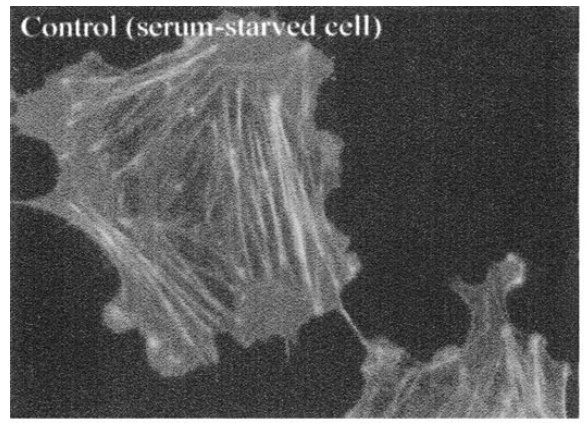

B

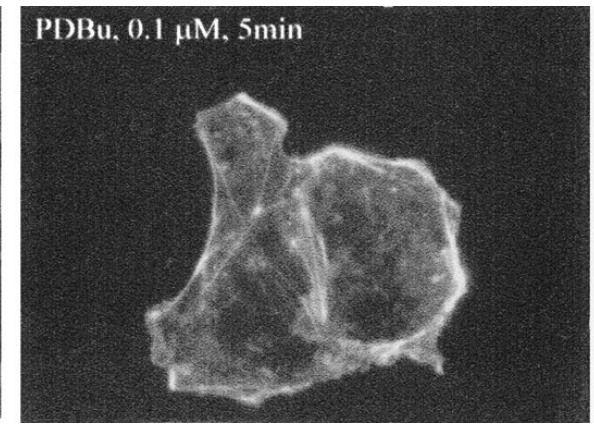



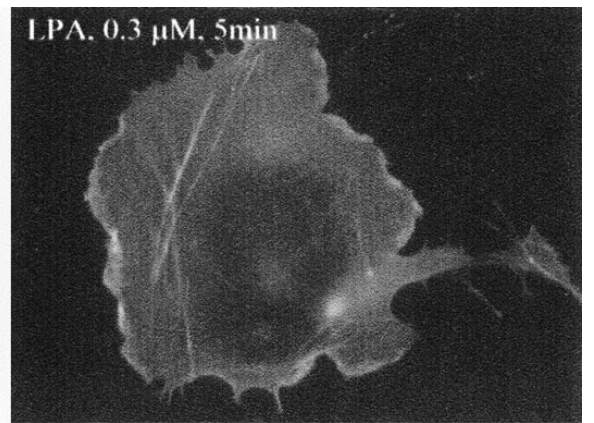

Fig. 4. F-actin bundle structures in NT-2 cells treated without (A) or with $0.1 \mu \mathrm{M}$ PDBu for 5 min (B) and $0.3 \mu \mathrm{M}$ LPA for $5 \min (\mathrm{C})$. 


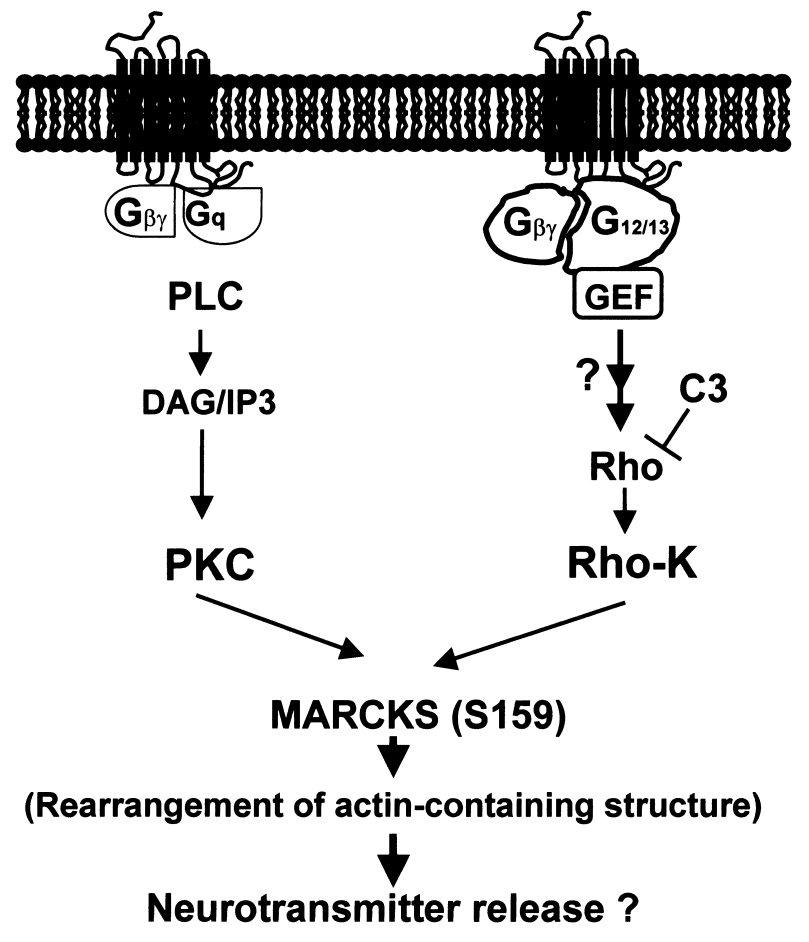

Fig. 5. Assumptive scheme for the receptors of LPA and the down-stream reactions that are involved in the activation of Rhokinase or/and PKC followed by MARCKS phosphorylation. DAG, diacylglycerol; IP3, inositol 1,4,5-triphosphate; GEF, guanine nucleotide exchange factor.

and subsequently reorganization of the F-actin bundle structure, and these events result in the trafficking of reserved vesicles to the active zone. It is known that some kinds of neuronal stimulants such as inflammation- and brain ischemia-related substances can activate the Rho/Rho-kinase pathway through an activation of trimeric G-protein. It is possibly that some of these stimulants evoke neurotransmitter release through the phosphorylation of MARCKS by Rho-kinase; however, the molecular mechanisms are not precisely elucidated. These concepts may allow us understand a novel mechanism of neurotransmitter release evoked by inflammation- and brain ischemia-related substances, LPA, angiotensin II, thrombin etc. $(4,10)$.

\section{References}

1 Sudhof TC. The synaptic vesicle cycle revisited. Neuron. 2000;28:317-320.

2 Dekker LV, De Graan PN, Gispen WH. Transmitter release: target of regulation by protein kinase C? Prog Brain Res. 1991;89:209-233.

3 Goodall AR, Turner NA, Walker JH, Ball SG, Vaughan PF. Activation of protein kinase $\mathrm{C}$-alpha and translocation of the myristoylated alanine-rich C-kinase substrate correlate with phorbol ester-enhanced noradrenaline release from SH-SY5Y human neuroblastoma cells. J Neurochem. 1997;68:392-401.

4 Neltner BS, Zhao Y, Sacks DB, Davis HW. Thrombin-induced phosphorylation of MARCKS does not alter its interactions with calmodulin or actin. Cell Signalling. 2000;12:71-79.

5 Elzagallai A, Rose SD, Brandan NC, Trifaro J-M. Myristoylated alanine-rich $\mathrm{C}$ kinase substrate phosphorylation is involved in thrombin-induced serotonin release from platelets. $\mathrm{Br} \mathrm{J}$ Haematol. 2001;112:593-602.

6 Lonart G, Sudhof TC. Assembly of SNARE core complexes prior to neurotransmitter release sets the readily releasable pool of synaptic vesicles. J Biol Chem. 2000;275:27703-27707.

7 Trifaro J-M, Rose SD, Lejen T, Elzagallaai A. Two pathways control chromaffin cell cortical F-actin dynamics during exocytosis. Biochimie. 2000;82:339-352.

8 Hartness ME, Wade JA, Walker JH, Vaughan PF. Overexpression of the myristoylated alanine-rich $\mathrm{C}$ kinase substrate decreases uptake and $\mathrm{K}^{+}$-evoked release of noradrenaline in the human neuroblastoma SH-SY5Y. Eur J Neurosci. 2001;13:925934.

9 Trifaro J-M, Vitale ML. Cytoskeleton dynamics during neurotransmitter release. Trends Neurosci. 1993;16:466-472.

10 Ikenoya M, Hidaka H, Hosoya T, Suzuki M, Yamamoto N, Sasaki Y. Inhibition of Rho-kinase-induced myristoylated alanine-rich $\mathrm{C}$ kinase substrate (MARCKS) phosphorylation in human neuronal cells by H-1152, a novel and specific Rhokinase inhibitor. J Neurochem. 2002;81:9-16.

11 Nagumo H, Ikenoya M, Sakurada K, et al. Rho-associated kinase phosphorylates MARCKS in human neuronal cells. Biochem Biophys Res Commun. 2001;280:605-609.

12 Moolenaar WH, Kraneburg O, Postma FR, Zondag GCM. Lysophosphatidic acid: G-protein signalling and cellular responses. Curr Opin Cell Biol. 1997;9:168-173.

13 Shiono S, Kawamoto K, Yoshida N, Kondo T, Inagami T. Neurotransmitter release from lysophosphatidic acid stimulated PC12 cells: involvement of lysophosphatidic acid receptors. Biochem Biophys Res Commun. 1993;193:667-673.

14 Molderings GJ, Bonisch H, Hammermann R, Gothert M, Bruss M. Noradrenaline release inhibiting receptors on PC12 cells devoid of $\alpha_{2}$ and $\mathrm{CB}_{1}$ receptors: similarities to presynaptic imidzoline and edg receptors. Neurochem Int. 2002;40:157-167.

15 Takuwa Y, Takuwa N, Sugimoto N. The Edg family G proteincoupled receptors for lysophospholipids: their signaling properties and biological activities. J Biochem. 2002;131:767-771.

16 Yamauchi E, Kiyonami R, Kanai M, Taniguchi H. The Cterminal conserved domain of MARCKS in phosphorylated in vivo by proline-directed protein kinase. Application of ion trap mass spectrometry to the determination of protein phosphorylation sites. J Biol Chem. 1998;273:4367-4371.

17 Taniguchi H, Manenti S, Suzuki M, Titani K. Myristoylated alanine-rich $\mathrm{C}$ kinase substrate (MARCKS), a major protein kinase $\mathrm{C}$ substrate, is an in vivo substrate of proline-directed protein kinase(s). A mass spectroscopic analysis of the posttranslational modifications. J Biol Chem. 1994;269:1829918302.

18 Ito K, Shimomura E, Iwanaga T, et al. Essential role of rho kinase in the $\mathrm{Ca}^{2+}$ sensitization of prostaglandin $\mathrm{F} 2 \alpha$-induced contraction of rabbit aortae. J Physiol (Lond). 2003;546:823836.

19 Kitazawa T, Eto M, Woodsome TP, Brautigan DL. Agonists 
trigger $\mathrm{G}$ protein-mediated activation of the CPI-17 inhibitor phosphoprotein of myosin light chain phosphatase to enhance vascular smooth muscle contractility. J Biol Chem. 2000;275: 9897-9900.

20 Chihara K, Amano M, Nakamura N, et al. Cytoskeletal rearrangements and transcriptional activation of c-fos regum response element by Rho-kinase. J Biol Chem. 1997;272:2512125127.

21 Hartwig JH, Thelen M, Rosen A, Janmey PA, Nairn AC, Aderem A. MARCKS is an actin filament crosslinking protein regulated by protein kinase $\mathrm{C}$ and calcium-calmodulin. Nature. 1992;356:618-622.

22 Yarmola EG, Edison AS, Lenox RH, Bubb MR. Actin filament cross-linking by MARCKS. Characterization of two actin-binding sites within the phosphorylation site domain. J Biol Chem. 2001;276:22351-22358.

23 Arbuzova A, Schmitz AAP, Vergeres G. Cross-talk unfolded: MARCKS proteins. Biochem J. 2002;362:1-12.

24 Aderem A. The MARCKS brothers: a family of protein kinase C substrates. Cell. 1992;71:713-716.

25 Blackshear PJ. The MARCKS family of cellular protein kinase C substrates. J Biol Chem. 1993;268:1501-1504.

26 Ouimet CC, Wang JK, Walaas SI, Albert KA, Greengard P. Localization of the MARCKS $(87 \mathrm{kDa})$ protein, a major specific substrate for protein kinase C, in rat brain. J Neurosci. 1990; 10:1683-1698.

27 Ohmori S, Sakai N, Shirai Y, et al. Importance of protein kinase $\mathrm{C}$ targeting for the phosphorylation of its substrate, myristoylated alanine-rich C-kinase substrate. J Biol Chem. 2000;275: 26449-26457.

28 Wang J, Arbuzova A, Hangyas-Mihalyne G, McLaughlin S. The effect domain of myristoylated alanine-rich C-kinase substrate binds strongly to phosphatidylinositol 4,5-bisphosphate. J Biol Chem. 2000;276:5012-5019.

29 Rose SD, Lejen T, Zhang L, Trifaro JM. Chromaffin cell F-actin disassembly and potentiation of catecholamine release in response to protein kinase $\mathrm{C}$ activation by phorbol esters is mediated through myristoylated alanine-rich $\mathrm{C}$ kinase substrate phosphorylation. J Biol Chem. 2001;276:36757-36763.

30 Vitale ML, Seward EP, Trifaro JM. Chromaffin cell cortical actin network dynamics control the size of the release-ready vesicle pool and the initial rate of exocytosis. Neuron. 1995; 14:353-363.

31 Yang H, Wang X, Sumners C, Raizada MK. Obrigatory role of protein kinase C-beta and MARCKS in vesicular trafficking in living neurons. Hypertension. 2002;39:567-572.

32 Stevens CF, Sullivan JM. Regulation of the ready releasable vesicle pool by protein kinase C. Neuron. 1998;21:885-893.

33 Polzin A, Shipitsin M, Goi T, Feig LA. Ral-GTPase influences the regulation of the readily releasable pool of synaptic vesicles. Mol Cell Biol. 2002;22:1714-1722.

34 Majewski H, Iannazzo L. Protein kinase C: a physiological mediator of enhanced transmitter output. Prog Neurobiol. 1998;55:463-475.

35 Hilfiker S, Augustine GJ. Regulation of synaptic vesicle fusion by protein kinase C. J Physiol. 1999;515:1.

36 Iwasaki S, Kataoka M, Sekiguchi M, Shimazaki Y, Sato K, Takahashi M. Two distinct mechanisms underlie the stimulation of neurotransmitter release by phorbol esters in clonal rat pheochromocytoma PC12 cells. J Biochem. 2000;128:407-414.

37 Neltner S, Zhao Y, Sacks DB, Davis HW. Thrombin-induced phosphorylation of MARCKS does not alter its interaction with calmodulin or actin. Cell Signal. 2000;12:71-79. 